\title{
Associations Between Gut Microbiota and Asthma Endotypes: A Cross-Sectional Study in South China Based on Patients with Newly Diagnosed Asthma
}

\author{
Xiao-Ling Zou $\mathbb{1}^{1, *}$ \\ Jin-Jie $\mathrm{Wu}^{2}$,* \\ Hui-Xia $\mathrm{Ye}^{3, *}$ \\ Ding-Yun Feng $\mathbb{D}^{1, *}$ \\ Ping Meng' \\ Hai-Ling Yang \\ Wen-Bin Wu (iD) \\ Hong-Tao Li ${ }^{1}$ \\ Zhen $\mathrm{He}^{2}$ \\ Tian-Tuo Zhang (D) \\ 'Department of Pulmonary and Critical \\ Care Medicine, The Third Affiliated \\ Hospital of Sun Yat-sen University, Institute \\ of Respiratory Diseases of Sun Yat-sen \\ University, Guangzhou, People's Republic of \\ China; ${ }^{2}$ Department of Colorectal Surgery, \\ The Sixth Affiliated Hospital of Sun Yat-sen \\ University, Guangzhou, People's Republic of \\ China; ${ }^{3}$ Department of Gynecology, The \\ Third Affiliated Hospital of Sun Yat-sen \\ University, Guangzhou, People's Republic of \\ China
}

*These authors contributed equally to this work

Correspondence: Tian-Tuo Zhang Department of Pulmonary and Critical

Care Medicine, The Third Affiliated

Hospital of Sun Yat-sen University,

Institute of Respiratory Diseases of Sun

Yat-sen University, 600 Tianhe Road,

Guangzhou, 510630, People's Republic of

China

Email zhtituli@I63.com

Zhen $\mathrm{He}$

Department of Colorectal Surgery, The Sixth Affiliated Hospital of Sun Yat-sen University, Guangzhou, 510655,

Guangdong, People's Republic of China

Email hezh5@mail.sysu.edu.cn
Objective: This study aimed to investigate the gut microbiome profile in different inflammatory phenotypes of treatment-naive newly diagnosed asthmatic adults, to gain insight on the associations between intestinal microbiota and phenotypic features that characterize asthma heterogeneity to develop new treatments for asthma.

Methods: Fresh stool samples were obtained from 20 healthy subjects and 47 newly diagnosed asthmatic patients prior to any interventions. The asthmatics were divided into allergic and non-allergic cohorts. Intestinal microbiota was analyzed by $16 \mathrm{~S}$ rRNA nextgeneration sequencing. Demographic and clinical parameters were collected. Alpha and beta diversity analysis were calculated to detect differences within sample phylotype richness and evenness between controls and asthmatic patients. Statistically significant differences between samples were analyzed for all used metrics, and features of gut bacterial community structure were evaluated in relation to extensive clinical characteristics of asthmatic patients. Results: Gut microbial compositions were significantly different between asthmatic and healthy groups. Alpha-diversity of the gut microbiome was significantly lower in asthmatics than in controls. The microbiome between allergic and non-allergic asthmatic patients were also different, and 28 differential species were identified. PPAR signaling pathway, carotenoid biosynthesis, and flavonoid biosynthesis were significantly positively correlated with allergy-associated clinical index, including FENO value, blood eosinophil counts, and serum IgE and IL-4 levels. A combination of Ruminococcus bromii, Brevundimonas vesicularis, and Clostridium disporicum showed an AUC of 0.743 in the specific allergic/non-allergic cohort. When integrating $C$. disporicum, flavone, flavonol biosynthesis, and serum IL-4 values, the AUC achieved 0.929 to classify asthmatics. At the same time, C. colinum and its associated functional pathway exhibited an AUC of 0.78 to distinguish allergic asthmatics from those without allergies.

Conclusion: We demonstrated a distinct taxonomic composition of gut microbiota in different asthmatic phenotypes, highlighting their significant relationships. Our study may support considerations of intestinal microbial signatures in delineating asthma phenotypes.

Keywords: asthma, phenotypes, gut microbiota, 16S rRNA next-generation sequencing, functional pathway

\section{Background}

Asthma is one of the most common chronic respiratory diseases that has affected more than 600 million people and caused 250,000 deaths each year. ${ }^{1}$ In addition, its pathogenesis is heterogeneous and includes multiple phenotypes with diverging clinical characteristics and outcomes. ${ }^{2}$ Asthmatic patients with different phenotypes 
show a distinct, sometimes even completely refractory, response to standard inhaled corticosteroid (ICS) therapy and require a large amount of resources for management. ${ }^{3}$ Allergic asthma is a highly prevalent phenotype of asthma displaying at least one form of allergy, usually characterized by type 2 (T2) inflammation ${ }^{4}$ defined by a high expression of cytokines interleukin-4 (IL-4), IL-5, and IL-13 in the serum, usually accompanied by elevated blood eosinophil (EOS) and immunoglobulin E (IgE) level. ${ }^{5}$ Most patients with "T2-high" inflammation respond well to ICS therapy. In contrast, those who respond poorly to ICS treatment have "corticosteroid resistance" and may progress to "refractory asthma". 6 The heterogeneous nature of asthma increases the disease's burden and challenges for appropriate diagnosis and therapy. Therefore, improved understanding of the biological drivers underlying different endotypes will advance more precise approaches to define asthma and determine the best treatment options.

The "hygiene hypothesis" was the first to suggest a link between microbes and allergy. ${ }^{7}$ Recently, the development of DNA/RNA sequencing technologies for microorganism identification has made more in-depth microbiome research possible. To date, growing evidence have revealed associations between bacterial composition and asthma. The different composition of lung microbiome between asthmatic and healthy individuals suggest that bacteria might contribute to the initiation of asthma ${ }^{8-10}$ and to the immune responses for microbiota residing in other sites, such as the gut. This has led to the concept of the "gut-lung axis," the dysbiosis of gut microbiota is linked to airway disease through modification of the immune response. ${ }^{11}$ The human gut in healthy subjects is colonized by $10^{14}$ bacteria and contains more than 1000 bacterial species. The interaction of the human gut and its microbiome has been conditioned by dietary and environmental variations, leading to various bacteria interacting both for defense and nutritional advantages. ${ }^{12}$

The potential for intestinal microbiota to modulate allergic airway inflammation has been demonstrated in a murine model. ${ }^{13}$ Altered gut microbiota of fetal age in mice has been reported to augment allergic sensitization. ${ }^{14}$ In humans, several studies have linked a dysbiosis of the gut microbiome early in life with an enhanced risk for asthma development later in life. ${ }^{15-17}$ Moreover, in clinical studies, the relationships between microbial dysbiosis and asthma phenotypes have predominantly been explored among adults whose asthma heterogeneity is well recognized. Adults with allergic asthma have a higher concentration of histamine-secreting bacteria in their gut than healthy subjects, suggesting a microbial source of histamine that affects manifestations of allergic asthma. ${ }^{18}$ Intestinal microbiota differences were also associated with sensitization to many aeroallergens and with differences in lung function among asthmatic adults. ${ }^{19}$ However, as the asthmatic patients included in the studies mentioned above had begun to use corticosteroids prior to the study period, it is unclear whether this difference in bacterial composition is a characteristic of asthma itself, related to corticosteroid use, or both. As asthma disease by itself, different phenotypes and medications (including corticosteroids) can significantly shape the microbiome profiles. Hence, these early findings invite a closer look into possible mechanisms by which the intestinal microbiota may influence features related to different endotypes of asthma in adult populations.

In this study, we investigated the composition of intestinal microbiota in newly diagnosed adults with asthma (had not received medication therapy) with different phenotypes and healthy controls from South China, analyzed their diversity at a taxonomic level, and explored the interaction of the dominant flora, to confirm the hypothesis that the characteristics of gut microbiome would be different between different inflammatory phenotypes of asthma. Additionally, we further investigated the relationships between the profile of gut bacterial flora and clinical parameters, such as forced expiratory volume in one second (FEV1), a fraction of exhaled nitric oxide (FENO) values, and IgE levels. Our results may help later diagnose different phenotypes of asthma better and allow us to use the microbiome as a biomarker in precision medicine approaches for the disease.

\section{Methods}

\section{Study Subjects and Sample Collection}

A total of 67 adults were recruited in this cross-sectional study from July 2020 to December 2020 in the ThirdAffiliated Hospital of Sun Yat-Sen University, China. Of them, 47 were newly diagnosed with bronchial asthma (treatment-naive) according to Global Initiative for Asthma 2019 guidelines, and the other 20 were healthy individuals (controls). All the participants were aged 1850 years. The exclusion criteria were as follows: abnormal body mass index (BMI $>28$ or $<18.5$ ); unusual dietetic regimen; smokers; history of colon cancer; autoimmune 
diseases; inflammatory bowel disease; diarrhea 4 weeks before taking the stool sample; antibiotic, probiotic, or vitamin administration less than 1 month before fecal sampling; those who received immunosuppressive therapy and/or allergen-specific immunotherapy in the last 5 years; and those on oral or inhaled glucocorticoid treatment during 6 months prior to the sample collection date. Clinical parameters of all the participants were collected. This study was conducted in accordance with the Declaration of Helsinki and approved by the institutional review boards of the Third-Affiliated Hospital of Sun Yat-sen University. Written informed consents were obtained from all individuals. Fresh stools were collected in sterile collection tubes and immediately transported and stored at $-80^{\circ} \mathrm{C}$, as previously described. ${ }^{19}$

\section{I6S rRNA Gene Sequencing and Microbiome Data Analysis}

Fecal bacterial DNA of $100 \mathrm{mg}$ fecal material was extracted by a manufacturer's instructions of the FastDNA Spin Kit for Feces. The DNA was diluted to $1 \mathrm{ng} / \mu \mathrm{L}$ across the sample set. The variable region $\mathrm{V} 4$ encoded for the $16 \mathrm{~S}$ rRNA gene was amplified using bacteria primer 515F/806R. PCR products operate electrophoresis on $2 \%$ agarose gel for qualification. Sequencing libraries were generated using TruSeq ${ }^{\circledR}$ DNA PCR-Free Sample Preparation Kit (Illumina, USA) and subjected to sequencing on an Illumina NovaSeq platform, and $250 \mathrm{bp}$ paired-end reads were generated. Chimeric sequences were detected and filtered to obtain the effective tags using the UCHIME algorithm. Data were analyzed with the software "Quantitative Insights into Microbial Ecology." Assembled sequences were clustered into operational taxonomic units (OTUs), picking at 97\% sequence identity using UPARSE. The clustered OTUs were classified against Greengenes Databases (Release 13.8). ${ }^{20,21}$

Alpha and beta diversity analyses were calculated. Principal component analysis (PCA) was performed using the ade4 package in the $\mathrm{R}$ platform (version 4.0.2). Partial Least Squares Discriminant Analysis (PLS-DA) was used to discriminate the clustering effect of different phenotypes. To assess the proportion of microbiome variance explained by different clinical factors, the adonis function of $\mathrm{R}$ package vegan was used to estimate the "one-to-all" effect size (R2). The area under the receiver operating characteristic curve (AUC/ROC) was used to appraise the disease prediction. In addition, the symbiotic relationship between microbiota was displayed using Cytoscape (v3.8.1) according to Spearman's rank correlation coefficient. Functional analysis was predicated with the Phylogenetic Investigation of Communities by Reconstruction of Unobserved States (PICRUSt) based on the high-quality tags of the microbiota. ${ }^{15}$

\section{Random Forest Model}

The predictability of the microbiome to disease stratification was estimated using a random forest package. The Random forests (RF) model tried to grow multiple decision trees with different samples and different initial variables to produce a strong classifier. Lists of taxa ranked by $\mathrm{RF}$ in order of importance of characteristics were determined over 100 iterations. The number of marker taxa was identified using 10-fold crossvalidation implemented with the function in the $\mathrm{R}$ package $\mathrm{RF}$ with five repeats. ${ }^{22}$ The accuracy of the model was examined using a ROC curve.

\section{LASSO Logistic Regression Model}

LASSO logistic regression model was constructed by glmnet package in R. Models were validated by 10 -fold stratified cross-validation testing. Lambda.min returned the value of $\lambda$ that gives minimum mean cross-validated error. Based on the value of Lambda.min, the most relevant microbiome units were obtained in the detection of asthma. The accuracy of the model was examined using a ROC curve.

\section{Measurement of Serum IgE and Cytokine IL-4 Levels}

Total serum and allergen-specific serum IgE levels were examined based on a previous study. ${ }^{15}$ Total serum IgE level was measured by ImmunoCAP (Phadia), and specific IgE levels to food (egg protein, milk, peanut, soybean, wheat, buckwheat, cashew nut, pistachio nuts, filbert, beef, mutton, shrimp, crab, peach, apple, mango, and strawberry) and inhalant (Dermatophagoides pteronyssinus and Dermatophagoides farinae) allergens were determined using a commercial assay for IgE (ImmunoCAP Phadiatop Infant; Phadia). Serum cytokine IL-4 level was measured according to the manufacturer's instructions.

\section{Bioinformatical and Statistical Analysis}

All statistical analyses were performed using Prism 8 (GraphPad Software, San Diego, CA) unless otherwise stated in individual method sections above. 
Differences between the two groups were evaluated using the Student's $t$-test (parametric) or Mann-Whitney $U$-test (non-parametric). For comparisons of more than 3 groups, one-way ANOVA (parametric) or Kruskal-Wallis test (nonparametric) were performed. Differences of adjusted $p<$ 0.05 were considered significant $\left({ }^{*} p<0.05 ; *^{*} p<0.01\right)$.

\section{Results}

\section{Demographic and Clinical Characteristics of Study Subjects}

Subjects were considered as allergic if they had evidence of allergic sensitization to one or more allergens. Of all the asthmatic patients, 27 were considered to be allergic. None of the healthy controls showed evidence of allergy. Clinical parameters and asthmatic characteristics were shown in Table 1. FENO values, serum IgE and IL-4 levels were significantly higher in asthmatic patients than healthy control subjects. At the same time, FEV1/ FEV1prediction (FEV1\%pred) and FEV1/Forced vital capacity (FEV1/FVC) ratio were significantly lower in asthmatic subjects.

\section{Characterization of the Gut Microbiota in}

\section{Asthmatic Patients}

Two negative sequencing controls (feces-free blanks processed with the same sequencing procedure) were introduced as quality controls to exclude the contamination of our samples. As expected, no bacterial DNA was detected in quality controls. In addition, the rarefaction and the rank abundance curves were shown in Supplementary Figure $1 \mathrm{~A}$ and $\mathrm{B}$, indicating the rationality of sequencing data.

Permutational multivariate ANOVA (PERMANOVA) was performed to identify any clinical characteristics that affected gut microbiota. Demographic parameters such as age, gender, and BMI were not statistically different between asthmatic and healthy subjects. Instead, the disease status and the serum IL-4 level were two main reasons for the difference in intestinal microbiome between patients and controls (Supplementary Figure 1C). The overall microbial composition was significantly different between asthmatic and healthy groups (Adonis, $p=0.004$ ) (Figure 1A). We subsequently measured the

Table I Demographic and Clinical Characteristics of Study Subjects

\begin{tabular}{|c|c|c|c|c|c|c|}
\hline & \multirow{2}{*}{$\begin{array}{l}\text { Healthy } \\
(N=20)\end{array}$} & \multirow{2}{*}{$\begin{array}{l}\text { Asthma } \\
(N=47)\end{array}$} & \multirow[t]{2}{*}{$p$ value } & \multicolumn{3}{|c|}{ Asthma } \\
\hline & & & & $\begin{array}{c}\text { Non- } \\
\text { Allergy }(\mathrm{N}=20)\end{array}$ & Allergy $(\mathrm{N}=27)$ & $p$ value \\
\hline Age & $20.64 \pm 14.53$ & $39.91 \pm 16.02$ & 0.8604 & $42.7 \pm 17.44$ & $37.85 \pm 14.88$ & 0.3102 \\
\hline Gender & & & $>0.9999$ & & & 0.513 \\
\hline Male & 10 & 22 & & 12 & 10 & \\
\hline Female & 10 & 25 & & 8 & 17 & \\
\hline Height (cm) & $163.2 \pm 8.36$ & $161.3 \pm 7.96$ & 0.3812 & $160.2 \pm 9.20$ & $162.11 \pm 6.99$ & 0.442 \\
\hline Weight (kg) & $57.85 \pm 10.18$ & $60.2 \pm 12.87$ & 0.4709 & $58.48 \pm I I .46$ & $61.48 \pm 13.90$ & 0.4346 \\
\hline BMI $\left(\mathrm{kg} / \mathrm{m}^{2}\right)$ & $21.58 \pm 2.39$ & $23.09 \pm 4.42$ & 0.1554 & $22.74 \pm \mathrm{II} .46$ & $23.35 \pm 4.87$ & 0.6497 \\
\hline FEVI (L) & $3.2 \pm 0.47$ & $2.57 \pm 0.79$ & $0.0007^{*}$ & $2.49 \pm 0.83$ & $2.64 \pm 0.76$ & 0.5327 \\
\hline FEVI\%pred & $95.43 \pm 7.77$ & $87.60 \pm|4.9|$ & $0.0301 *$ & $87.46 \pm 13.24$ & $87.7 I \pm 16.28$ & 0.9559 \\
\hline FEVI/FVC & $90.565 \pm 3.94$ & $80.47 \pm 9.83$ & $<0.000 I^{*}$ & $78.61 \pm 10.50$ & $81.85 \pm 9.27$ & 0.2677 \\
\hline FENO (ppb) & $20.25 \pm 5.72$ & $38.87 \pm|4.4|$ & $<0.000 I^{*}$ & $31.3 \pm 10.16$ & $44.48 \pm 14.66$ & $0.0012^{*}$ \\
\hline EOS (*I0E9/L) & $0.3485 \pm 0.14$ & $0.42 \pm 0.33$ & 0.3473 & $0.174 \pm 0.10$ & $0.6 I \pm 0.33$ & $<0.000 I^{*}$ \\
\hline $\lg \mathrm{E}(\mathrm{IU} / \mathrm{mL})$ & $(0-48,27)$ & $(0-53 \mathrm{I}, \mathrm{III})$ & $0.0005^{*}$ & $(0-142,46)$ & $(56-53|, 2| 3)$ & $<0.000$ I* $^{*}$ \\
\hline IL-4 & $33.4 I \pm 5.78$ & $45.61 \pm 9.45$ & $<0.0001 *$ & $37.3 \pm 6.80$ & $51.77 \pm 5.62$ & $<0.000 I^{*}$ \\
\hline
\end{tabular}

Notes: Data were presented by median (range) or mean \pm standard deviation ( $x \pm s)$ (continuous). ${ }^{*} p<0.05$.

Abbreviations: BMI, Body Mass Index; FEVI, forced expiratory volume in one second; FEVI\%pred, FEVI/FEVI prediction; FVC, Forced vital capacity; FENO, Fractional exhaled nitric oxide; EOS, Eosinophil; IgE, Immunoglobulin E; IL-4, Interleukin-4. 

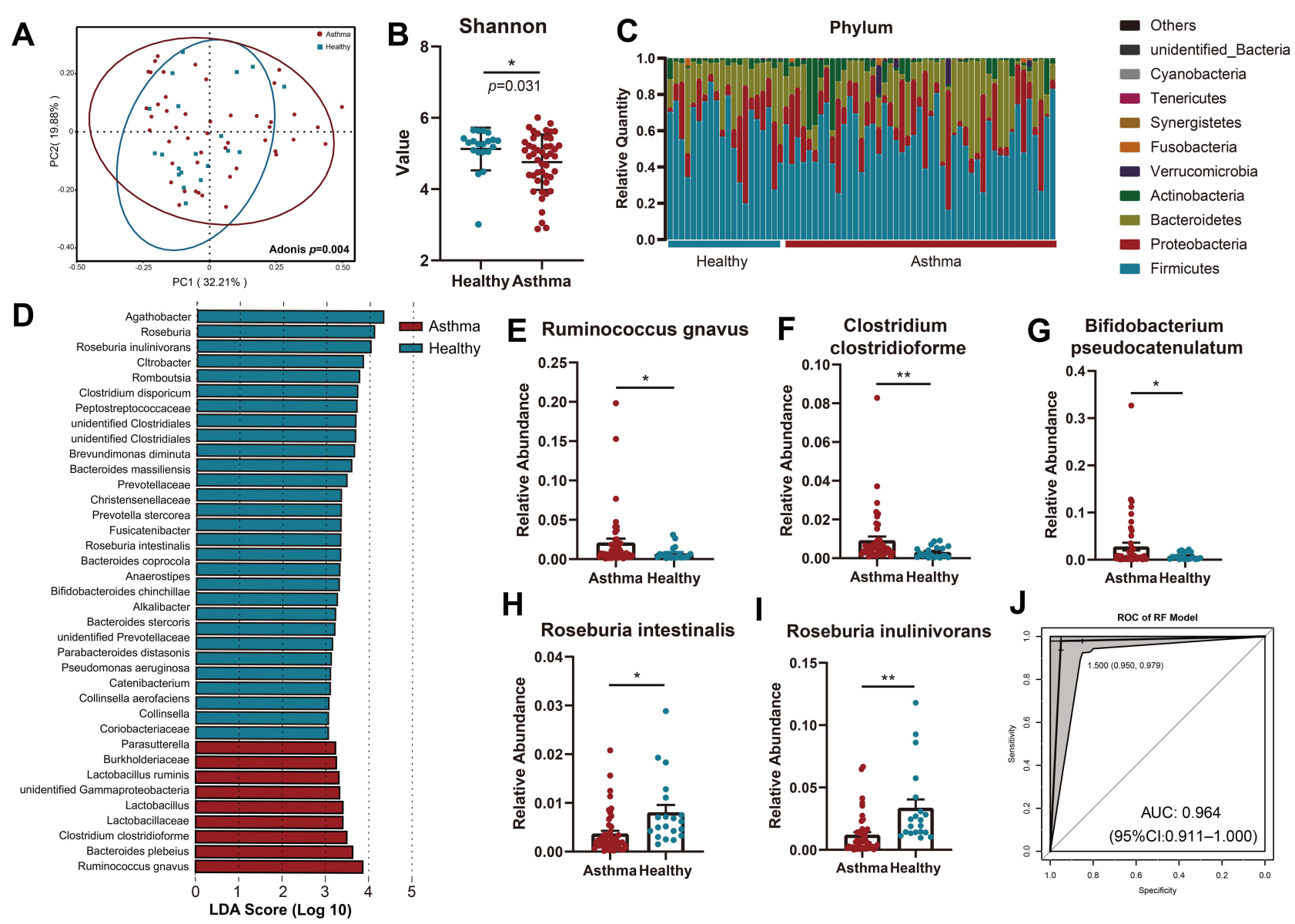

Figure I Characterization of the gut microbiota in asthmatic patients. (A) The overall microbial composition was different between asthmatic and healthy groups (Adonis, $p=0.004)$. (B) Alpha-diversity of the intestinal microbiome was lower in asthmatic patients compared to control subjects by using Shannon indices $(p=0.03 \mathrm{I})$. (C) General landscape of gut microbiome in asthmatic patients and control subjects. (D) Differentially abundant bacterial communities between asthmatic patients and healthy control subjects by using LEfSe. (E-I) Species significantly enriched in asthmatic patients (E-G) and healthy controls (H and $\mathbf{I})$ by using Student's $t$-test. $(\mathbf{J})$ ROC analysis of the bacterial biomarkers in RF model achieved an AUC of 0.964 to classify asthmatic patients from healthy controls. *, $p<0.05 ; * *, p<0.0$.

microbial diversity using Shannon indices and found that the alpha-diversity of asthmatic patients was significantly lower than those from control $(p=0.031)$ (Figure 1B).

We next assessed the general landscape of gut microbiome composition in all subjects and revealed the presence of similar communities (Figure 1C). Linear discriminant analysis of effect size showed that multiple species such as Ruminococcus gnavus, Bacteroides plebeius, and Clostridium clostridioforme were enriched in asthmatic patients while Roseburia inulinivorans and Clostridium disporicum were enriched in healthy controls (Figure 1D). To further clarify the microbial difference, we compared the bacterial at the species level. Species significantly enriched in asthmatic patients included Ruminococcus gnavus, Clostridium clostridioforme, and Bifidobacterium pseudocatenulatum (Figure 1E-G). Significantly depleted species included Roseburia intestinalis and Roseburia inulinivorans (Figure $1 \mathrm{H}$ and I).
In order to investigate the potential role of the gut microbiome, we constructed random-forest and LASSO logistic regression classifiers to discriminate asthma patients from healthy controls. The AUC/ROC achieved 0.964 for the Random-forest classifier (Figure 1J), while similar results were obtained by LASSO logistic regression classifier (Supplementary Figure 2).

\section{Differences of Intestinal Microbiome Between Different Asthma Phenotypes}

As expected, FENO values, blood EOS counts, serum IgE and IL-4 levels were higher in allergic asthmatic patients than non-allergic ones (Table 1). Although there were no significant differences in $\alpha$-diversity between allergic and non-allergic asthmatic patients (Supplementary Figure 3), we found an apparent difference in microbiome between them (Figure 2A). Twenty-eight differential species were 
A

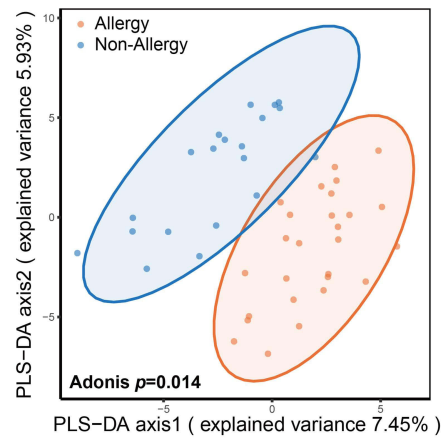

B

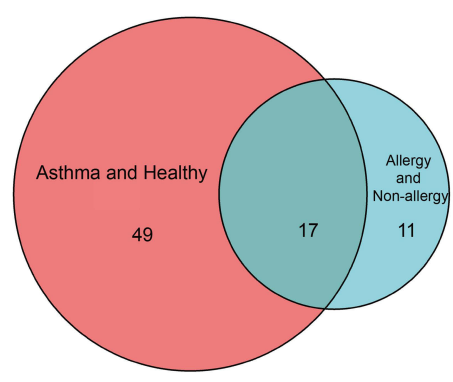

C

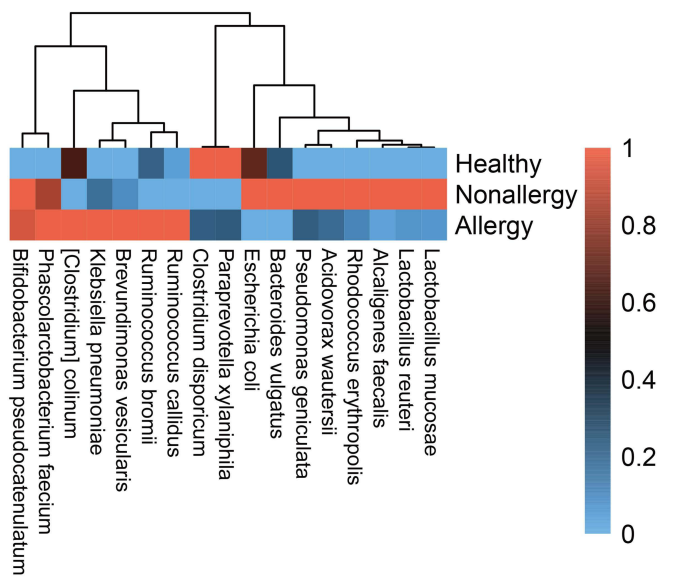

D
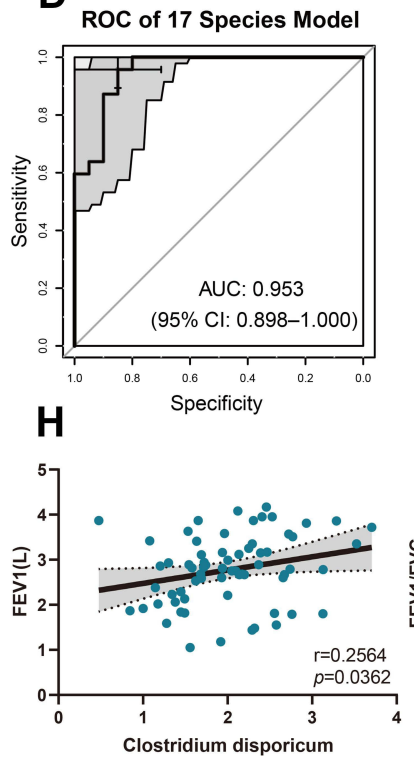

E
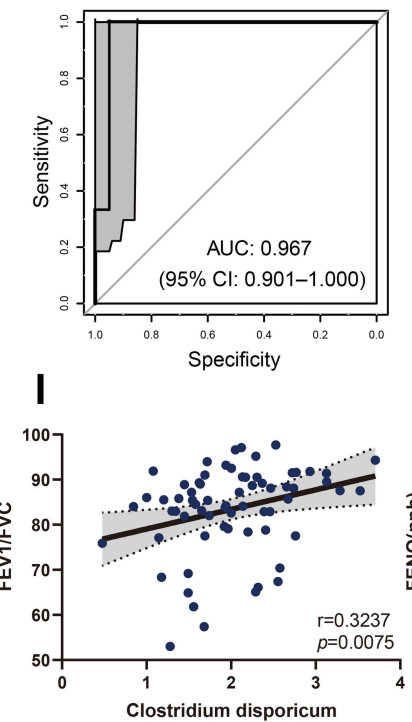

F ROC of 3 Species Model
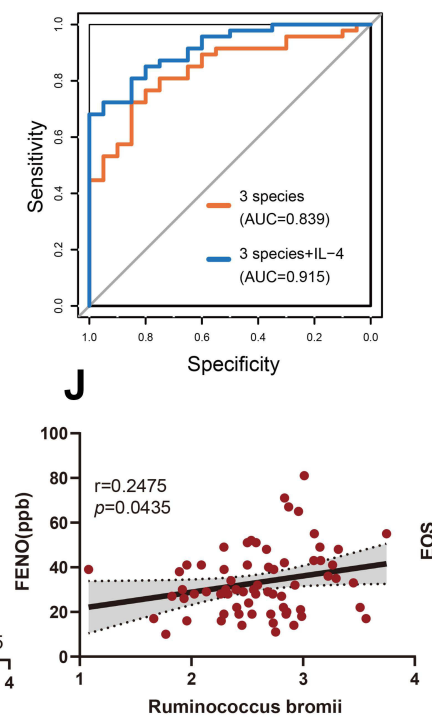

G ROC of 3 Species Model
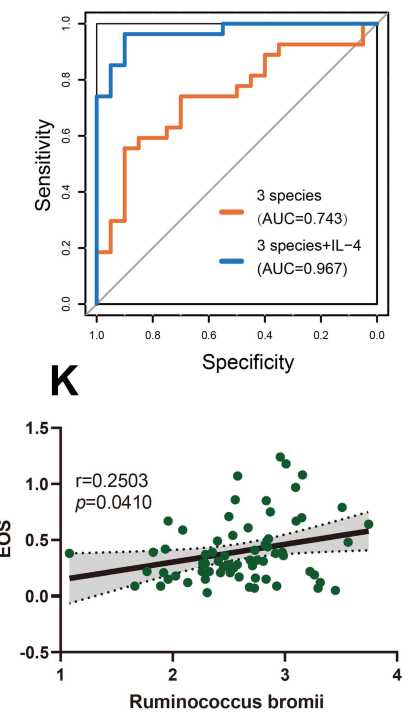

Figure 2 Differences of intestinal microbiome between different asthma endotypes. (A) PLS-DA of intestinal microbiome difference between allergic and non-allergic asthmatic patients (Adonis, $p=0.014$ ). (B and $\mathbf{C}$ ) Differential species of gut microbiome in allergic, non-allergic asthmatic patients, and healthy controls. ( $\mathbf{D}$ and $\mathbf{E})$ The AUC of 17 differential species reached 0.953 to predict asthma from healthy controls (D), and 0.967 to distinguish allergic/non-allergic cohort (E). (F and $\mathbf{G})$ Combination of Ruminococcus bromii, Brevundimonas vesicularis and Clostridium disporicum resulted in an AUC of 0.839 to discriminate healthy/asthma cohort (F) and 0.743 in allergic/nonallergic cohort (G). ( $\mathbf{H}$ and $\mathbf{I})$ Clostridium disporicum was positively correlated with FEVI $(r=0.2564, p=0.0362)(\mathbf{H})$ and FEVI/FVC ratio $(r=0.3237, p=0.0075)$, (I). $(\mathbf{J}$ and K) Ruminococcus bromii was positively correlated with FENO $(r=0.2475, p=0.0435)(\mathrm{J})$ and EOS level $(r=0.2503, p=0.04 \mathrm{I})(\mathbf{K})$.

identified, and 17 of these species were significantly different from those in healthy controls (Figure 2B and C). To establish a microbiotic signature for disease prediction, we tested the diagnostic potential of these 17 differential species in asthmatic stratification. It showed an AUC of 0.953 to detect a patient with asthma and 0.967 to stratify allergic/non-allergic asthma (Figure 2D and E). These data indicated the close association of gut microbiome and different status of the disease.

Specifically, a combination of Ruminococcus bromii (R. bromii), Brevundimonas vesicularis (B. vesicularis), and Clostridium disporicum (C. disporicum) resulted in an AUC of 0.839 in the healthy/asthma cohort
(Figure 2F) and 0.743 in allergic/non-allergic cohort (Figure 2G). As serum IL-4 level was significantly different among patients of three groups, we then tested its addition to the three-species signature. As expected, the AUC drastically increased to 0.915 to classify a patient with asthma (Figure 2F), while it improved to 0.967 in allergic/non-allergic stratification (Figure $2 \mathrm{G}$ ). Moreover, C. disporicum was significantly positively correlated with FEV1 $(\mathrm{r}=0.2564, p=0.0362)$ and FEV1/FVC ratio $(\mathrm{r}=0.3237, p=0.0075) \quad($ Figure $2 \mathrm{H}$ and $\mathrm{I})$. Meanwhile, $R$. bromii was significantly positively correlated with FENO $(\mathrm{r}=0.2475, p=0.0435)$ and blood EOS count $(\mathrm{r}=0.2503, p=0.041)$ (Figure $2 \mathrm{~J}$ and $\mathrm{K})$. These 

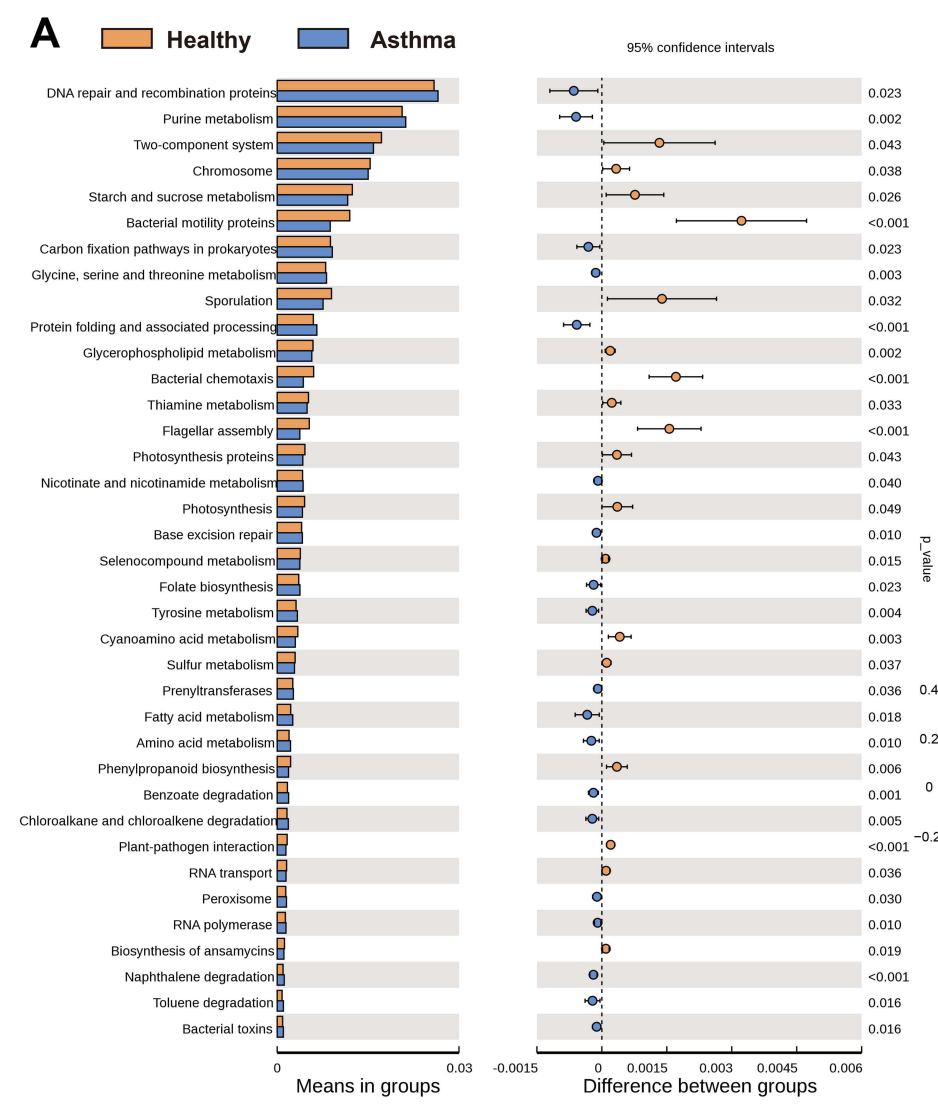

B

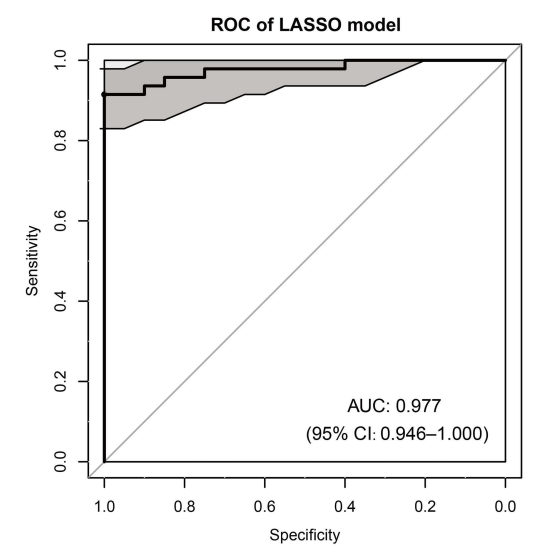

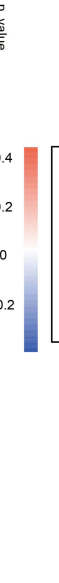

Figure 3 Functional analysis of gut microbiota and their association with asthma. (A) Differential pathways presented in asthmatic and control groups with PICRUSt analysis. (B) Model created by 16 microbiome-encoded pathways showed an AUC of 0.977 to distinguish asthma from healthy controls. (C) Correlation analysis between functional pathways and clinical indexes in asthmatic patients. *, $p<0.05 ; * *, p<0.01$.

data indicated the clinical significance of the gut microbiome in different statuses of asthma patients.

\section{Functional Analysis of Gut Microbiota and Their Association with Asthma}

We subsequently investigated functional changes within the gut microbiome between asthmatic patients and controls. Functional pathways were identified using HMP tool HUMAnN2 and annotated using KEGG, which indicated the involvement of metabolic pathways (Supplement Figure 4A). Further PICRUSt analysis also found that various metabolic pathways were enriched in the asthmatic group (Figure 3A). In contrast, some signal pathways such as bacterial motility proteins, bacterial chemotaxis, and flagellar assembly were down-regulated in the asthmatic group. We applied a LASSO logistic regression model to identify their clinical association, revealing an optimal set of 16 microbiome-encoded pathways. The AUC reached 0.977 in distinguishing asthma from controls (Figure 3B). Specifically, three pathways (PPAR signaling pathway, Carotenoid biosynthesis, and Flavonoid biosynthesis) were significantly positively correlated to allergy-associated clinical indexes, including FENO value, serum IgE/IL-4 level, and blood EOS count. Instead, D-Arginine and D-ornithine metabolism and mRNA surveillance pathway were both significantly negatively correlated with FEV1/FVC ratio (Figure 3C). However, the pathways bacterial chemotaxis did not exhibit any significant association with various clinical parameters. These data indicated the clinical significance of the microbial functional signals in the development of asthma.

\section{Distinct Gut Microbiotic Community to Mediate the Development of Different Asthma Phenotypes}

We finally integrated the microbiome, clinical indexes, and functional pathways for analysis to further depict the intestinal microbiotic community. It exhibited distinct characteristics of intestinal microbiome among different 
groups (Figure 4A and Supplementary Figure 4B). Moreover, $C$. disporicum was positively correlated with flavone and flavonol biosynthesis $(\mathrm{r}=0.3924, p=0.001)$ (Figure 4B). To explore the role of $C$. disporicum- flavone and flavonol biosynthesis axis in asthma, we evaluated their performance in disease stratification. It showed an AUC of 0.852 to distinguish asthma from controls (Figure 4C). When IL-4 was added, the AUC increased to 0.929 (Figure 4D).

Although $C$. disporicum was depleted in asthmatic patients, its role in subgroup classification was compromised, indicating the involvement of other bacteria in disease progression. As shown in Figure 4A and B, the role of Clostridium colinum (C. colinum) in asthmatic patients with allergic histories was different from those without allergic histories. Furthermore, C. colinum from allergic asthmatic patients was significantly negatively correlated with GPI anchor biosynthesis, mRNA surveillance pathway, and Indole alkaloid biosynthesis (Figure 4E). In contrast, C. colinum from non-allergic ones was significantly positively correlated with bacterial chemotaxis, PPAR signaling pathway, and Second bile acid biosynthesis, indicating the characteristics of the microbiome in different asthmatic phenotypes. Combining C. colinum and its associated pathways exhibited an AUC of 0.78 to stratify allergic asthmatic patients from those without allergy (Figure 4F).

\section{Discussion}

The present study profiled the feces bacteria compositions in newly diagnosed adult asthmatic patients from South China using the 16S rRNA gene sequencing approach. Our findings disclosed dysbiosis of intestinal microbiota makeups in asthmatic patients compared to healthy people. We evaluated the microbiome profiles in different inflammatory phenotypes of asthma and demonstrated a correlation between gut microbiota anomalies and different asthmaassociated phenotypes.

Lower bacterial phylotype richness and diversity were observed in feces from patients with asthma compared to healthy controls in this study. ${ }^{19,23}$ It was consistent with some previous reports but converse to the others. ${ }^{24,25}$ The differences between our results and others can be attributed to the different demographics and ages of the cohorts. Individuals originating from different populations might contribute to this variability because of exposure to different environments and risk factors. ${ }^{26,27}$ Other factors include multiple asthma phenotypes studied, use of corticosteroid or other medications, ${ }^{28}$ and different dietary habits. ${ }^{29}$ Moreover, the sample size is a huge determining factor that should be considered while designing the microbiome asthma studies.

To our knowledge, adult asthma is phenotypically heterogeneous, ${ }^{30}$ which is usually responsible for failure of asthma therapy. However, the mechanistic underpinnings of many asthma phenotypes remain poorly understood. Increasing research has shown that microbiota in the intestinal tract plays a critical role in the complex pathological process of different asthma phenotypes. ${ }^{18,31}$

The present study refined the list of bacterial groups previously associated with asthma to a more specific set of bacterial candidates associated with symptoms, such as allergy and Th2 inflammation. Apparent differences of microbiome between allergic and non-allergic asthmatics were observed, and three species (R. bromii, B. vesicularis, and $C$. disporicum) were indicated to be significantly associated with asthma phenotype. B. vesicularis has been found in the sputum of cystic fibrosis patients and used as potential bioremediation of polyaromatic hydrocarbons. ${ }^{32}$ Reduction of $R$. bromii in the gut was demonstrated to contribute to the occurrence of Crohn's disease. ${ }^{33}$ Neither of the two bacteria mentioned above has been reported to be associated with asthma phenotypes before. The present study was the first time to point out this. Moreover, $R$. bromii was significantly positively correlated with FENO and blood EOS level, indicating a harmful role in disease development, especially in allergic patients. C. disporicum, one of the first and most dominant bacterial species that colonize the intestines of healthy infants, has been found associated with gastrointestinal dysfunctions. ${ }^{16,33}$ Even though its role in asthma is unknown yet, its presence at the initiation of microbiome colonization in healthy infants led us to hypothesize that reducing $C$. disporicum positively affects asthma. Consistent with our hypothesis, the present study showed that $C$. disporicum was significantly positively correlated with FEV1 and FEV1/FVC ratio in asthmatic patients. Interestingly, though $C$. disporicum was significantly positively correlated with FEV1 and FEV1/FVC, it was not correlated with FENO levels, and likewise, only $R$. bromii was significantly positively correlated with FENO but not with FEV1 and FEV1/FVC. The possible reason may be that although changes in FEV1, FEV1/FVC ratio, and FENO levels usually go hand-in-hand, they do not always appear together in asthma. Spirometry is typically used to diagnose and characterize asthma, and change in \%FEV 1 


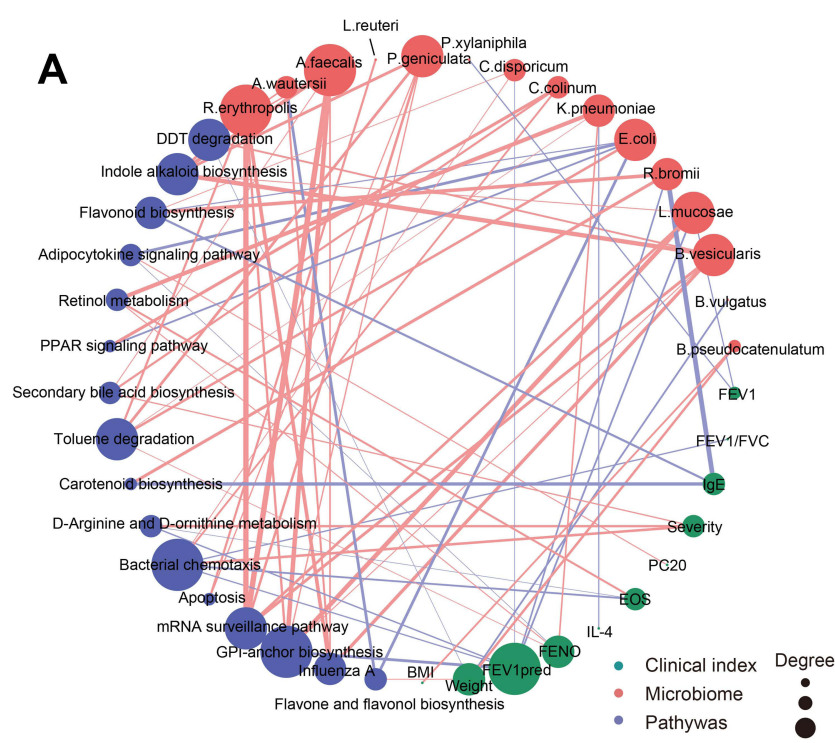

Non-allergic patients with asthma

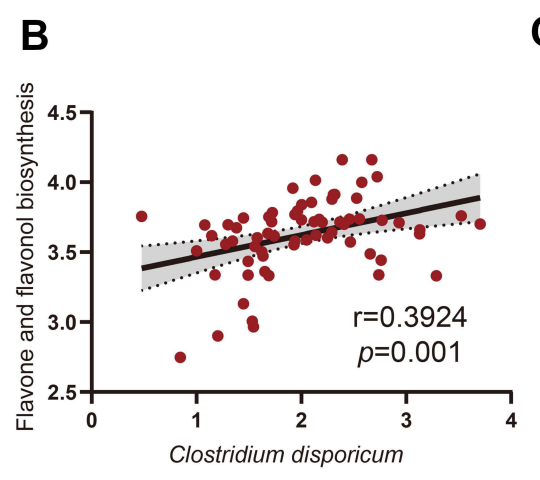

C

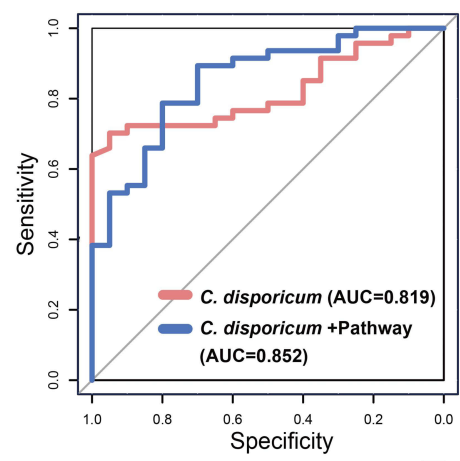

E

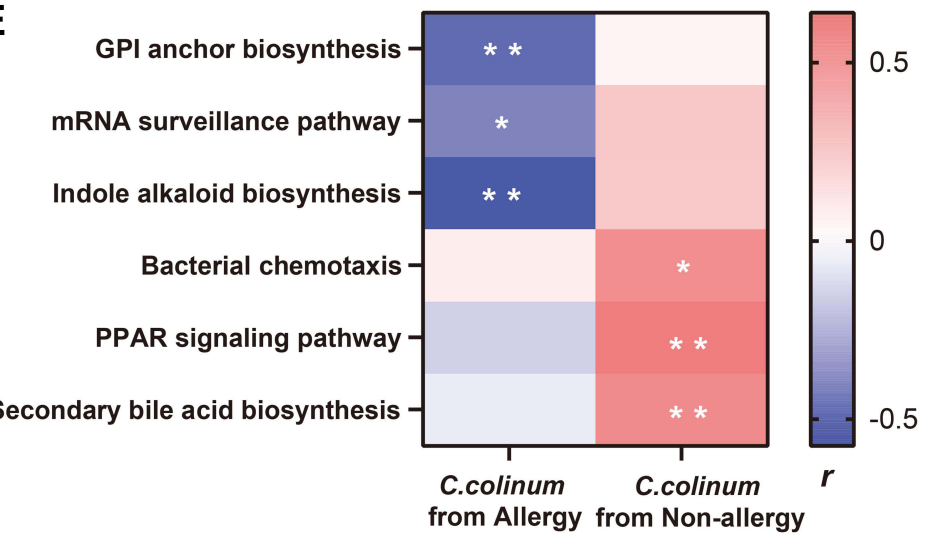

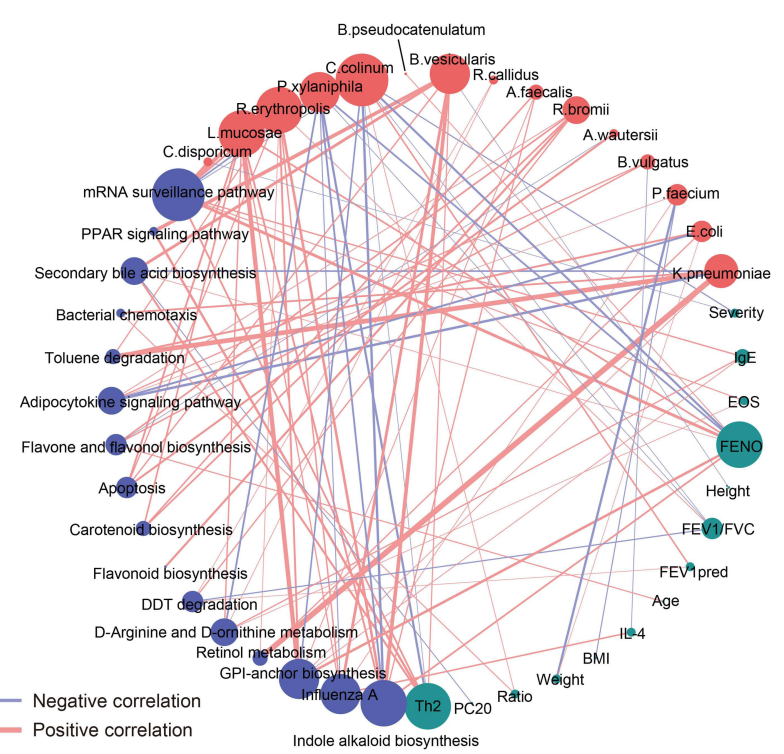

Allergic patients with asthma

D

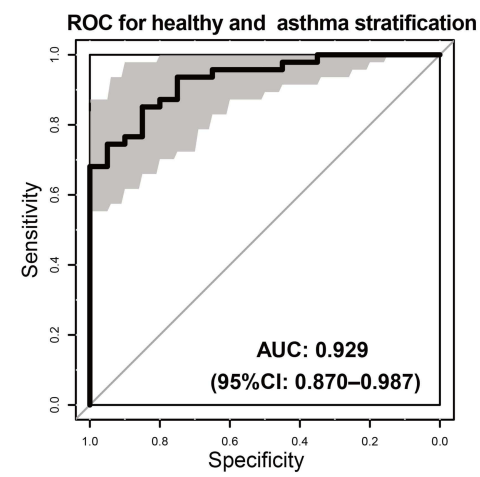

$\mathbf{F}$

ROC for allergic and non-allergic patients with asthma

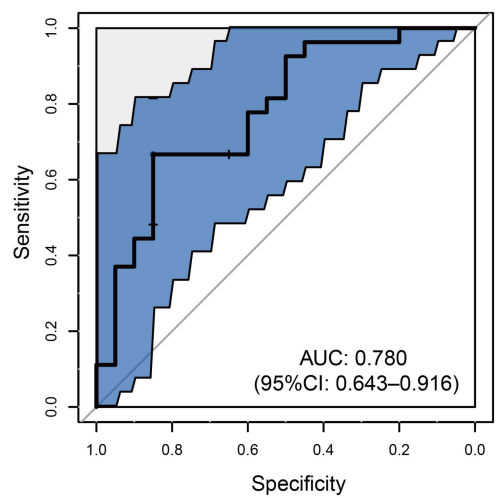

Figure 4 Distinct intestinal microbiotic community to mediate development of different asthma endotypes. (A) Integrated analysis of microbiome, clinical index and functional pathways in asthmatic patients with and without allergic history. (B) Correlation analysis between Clostridium disporicum (C. disporicum) and functional pathway of flavone and flavonol biosynthesis. (C) Clinical performance of $C$. disporicum and functional pathway for prediction of asthma. (D) Combination of $C$. disporicum, Flavonoid biosynthesis pathway and serum IL-4 level exhibited an AUC of 0.929 to classify asthmatic patients and healthy controls. (E) Correlation analysis between C. colinum and associated functional pathways. (F) Combination of $C$. colinum and its associated functional pathways resulted in an AUC of 0.780 to distinguish asthmatic patients with allergic history from non-allergic ones. *, $p<0.05 ; * *, p<0.01$.

can be used as part of risk assessment for asthma outcomes. ${ }^{34}$ Meanwhile, FENO correlates well with eosinophilic airway inflammation. But it is not present in all asthma patients, as eosinophilic bronchitis or a part of COPD also shows eosinophilic inflammation. ${ }^{35}$ Thus FENO is only used as an adjunct for asthma diagnosis. 
Change in FENO did not predict outcomes. ${ }^{36}$ Our finding indicated that $C$. disporicum alone was associated with asthma and potential to predict asthma, but not allergy status of asthmatic patients. These gut microbiota in select asthma phenotypes, coupled with these associations, suggest either a colonizing or a potential pathogenic role. Their difference in different phenotypes suggests that they might be useful, either alone or as part of a cluster of other biological or clinical markers, to delineate clinical asthma endotypes.

We further explored the potential correlations between bacteria-associated functions and clinical features with PICRUSt analysis. Durack et $\mathrm{al}^{37}$ demonstrated significant enrichment of amino acid and carbohydrate metabolism in atopic asthma. Another study identified significant relationships between bacteria-encoded functions and pathways. ${ }^{38}$ In this study, we also found that various metabolic pathways were enriched in the asthmatic group. The PPAR signaling pathway, Carotenoid biosynthesis, and Flavonoid biosynthesis were significantly positively correlated with FENO, serum IgE and IL-4, and blood EOS count. In contrast, flavone and flavonol biosynthesis was negatively correlated with these indexes. Peroxisome proliferator-activated receptors (PPARs) are nuclear hormone receptors important in cellular processes, including inflammation and immune response. It was considered a potential predictive and prognostic molecular marker in asthma. The PPAR signaling pathway was overrepresented in asthmatics, aligned with asthma susceptibility, and correlated with asthma risk and prognosis. ${ }^{39,40}$ Carotenoid biosynthesis is also involved in the pathogenesis of asthma. A higher concentration of a-carotene was suggested as evidence of increased risk of asthma in children by the age of 5 years. ${ }^{41,42}$ Recent reports have shown beneficial effects of flavonoids as anti-allergic and antiinflammatory through decreasing the incidence of asthma by inhibiting histamine release, synthesis of IL-4 and IL13, and CD40 ligand expression on basophils. ${ }^{43,44}$ Clinical research has suggested that Arg was negatively linked to airway hyperreactivity development and served as a determinant in the physiologic characteristics that typify human asthma. ${ }^{45,46} \mathrm{We}$ also found that D-Arginine metabolism might play protective roles in the development of asthma as they were both significantly negatively correlated with FEV1/FVC ratio. The results in our study were in agreement with previous studies that demonstrated a relationship between these pathways with asthma.
As mentioned above, a combination of $C$. disporicum and the flavone and flavonol biosynthesis axis together distinguished asthmatics from healthy controls. Hence, we subsequently integrated the gut microbiome, clinical index, and functional pathways to further identify the role of the microbiome in asthma. When IL-4 was added, it exhibited a better performance to classify asthmatic patients from controls than that only used microbiome or other signal indexes alone. In addition, adding of $C$. colinum and its associated pathways also performed well to stratify asthmatic patients with allergic history from those without allergy. Thus, this analysis integrated the clinical data, gut microbiome, and function to enhance the role of the microbiome in the pathogenesis of specific asthmatic phenotypes. It suggested potentially important relationships between gut microbiota functions and asthma phenotypes in established disease of adults.

Whereas there are still limitations in the present study. Firstly, our study was conducted at a single medical center located in South China. As a result, the demographic and other characteristics of subjects analyzed are more homogeneous due to the lack of differences on dietary intake and environmental pollution associated with asthma. Secondly, the sample size is relatively small, even though many key comparisons achieved statistical significance with this small dataset. Moreover, there are no allergic subjects among the healthy controls, which is a limitation to the analysis of the allergic subgroup compared to nonallergic asthmatics. Thirdly, gut microbiota and functional pathways to predict asthma phenotype were not verified in this study. Hence, further work is needed to validate our findings in larger multicenter cohorts from different regions and determine the mechanistic pathways involved.

In conclusion, our study demonstrates potential associations between gut microbiota and phenotypes in established asthma of treatment-naive adults. We also highlighted correlations between select predicted bacterial functional pathways and clinical parameters. Our findings provide potential new biomarkers that might be useful, particularly when combined with other clinical and biological markers, to distinguish asthma endotypes. It also suggests a possibility of more targeted therapies for asthma by modulating select bacterial genera.

\section{Abbreviations}

ICS, inhaled corticosteroid; IL, interleukin; DNA, deoxyribonucleic acid; RNA, ribonucleic acid; BMI, body mass index; OTUs, operational taxonomic units; PCA, principal 
component analysis; PLS-DA, Partial Least Squares Discriminant Analysis; AUC/ROC, area under the receiver operating characteristic curve; PICRUSt, Phylogenetic Investigation of Communities by Reconstruction of Unobserved States; RF, Random forests; ANOVA, analysis of variance; FEV1, forced expiratory volume in one second; FEV1\%pred, FEV1/FEV1 prediction; FVC, Forced vital capacity; FENO, Fractional exhaled nitric oxide; EOS, Eosinophil; IgE, Immunoglobulin E; PPAR, Peroxisome proliferator-activated receptors.

\section{Ethics Approval and Consent to Participate}

This study was approved by the Institutional Review Board of the Third-Affiliated Hospital of Sun Yat-sen University.

\section{Funding}

This study was funded by the National Natural Science Foundation of China (grant numbers 81970017 and 81973984).

\section{Disclosure}

The authors declare that they have no conflicts of interest related to this study.

\section{References}

1. Mathew J, Aronow WS, Chandy D. Therapeutic options for severe asthma. Arch Med Sci. 2012;8(4):589-597. doi:10.5114/aoms.2012. 30280

2. Borish L, Culp JA. Asthma: a syndrome composed of heterogeneous diseases. Ann Allergy Asthma Immunol. 2008;101(1):1-8;quiz 8-11, 50. doi:10.1016/S1081-1206(10)60826-5

3. Wenzel SE. Emergence of biomolecular pathways to define novel asthma phenotypes. Type-2 immunity and beyond. Am J Respir Cell Mol Biol. 2016;55(1):1-4. doi:10.1165/rcmb.2016-0141PS

4. Kozik AJ, Huang YJ. The microbiome in asthma: role in pathogenesis, phenotype, and response to treatment. Ann Allergy Asthma Immunol. 2019;122(3):270-275. doi:10.1016/j.anai.2018.12.005

5. Smith SG, Chen R, Kjarsgaard M, et al. Increased numbers of activated group 2 innate lymphoid cells in the airways of patients with severe asthma and persistent airway eosinophilia. J Allergy Clin Immunol. 2016;137(1):75-86.e8. doi:10.1016/j.jaci.2015.05.037

6. Robinson D, Humbert M, Buhl R, et al. Revisiting type 2-high and type 2-low airway inflammation in asthma: current knowledge and therapeutic implications. Clin Exp Allergy. 2017;47(2):161-175. doi:10.1111/cea. 12880

7. Stiemsma LT, Turvey SE. Asthma and the microbiome: defining the critical window in early life. Allergy Asthma Clin Immunol. 2017;13 (1):3. doi:10.1186/s13223-016-0173-6

8. Taylor SL, Leong LE, Choo JM, et al. Inflammatory phenotypes in patients with severe asthma are associated with distinct airway microbiology. J Allergy Clin Immunol. 2018;141(1):94-103.e15. doi:10.1016/j.jaci.2017.03.044
9. Yang X, Li H, Ma Q, Zhang Q, Wang C. Neutrophilic asthma is associated with increased airway bacterial burden and disordered community composition. Biomed Res Int. 2018;2018:9230234. doi: $10.1155 / 2018 / 9230234$

10. Li N, Qiu R, Yang Z, et al. Sputum microbiota in severe asthma patients: relationship to eosinophilic inflammation. Respir Med. 2017;131:192-198. doi:10.1016/j.rmed.2017.08.016

11. Penders J, Stobberingh EE, van den Brandt PA, Thijs C. The role of the intestinal microbiota in the development of atopic disorders. Allergy. 2007;62(11):1223-1236. doi:10.1111/j.1398-9995.2007.01 462.x

12. Bäckhed F, Ley RE, Sonnenburg JL, Peterson DA, Gordon JI. Hostbacterial mutualism in the human intestine. Science. 2005;307 (5717):1915-1920. doi:10.1126/science.1104816

13. Zaiss MM, Rapin A, Lebon L, et al. The intestinal microbiota contributes to the ability of helminths to modulate allergic inflammation. Immunity. 2015;43(5):998-1010. doi:10.1016/j. immuni.2015.09.012

14. Ottman N, Ruokolainen L, Suomalainen A, et al. Soi 1 exposure modifies the gut microbiota and supports immune tolerance in a mouse model. J Allergy Clin Immunol. 2019;143(3):1198-1206. e12. doi:10.1016/j.jaci.2018.06.024

15. Durack J, Kimes NE, Lin DL, et al. Delayed gut microbiota development in high-risk for asthma infants is temporarily modifiable by Lactobacillus supplementation. Nat Commun. 2018;9(1):707. doi:10. 1038/s41467-018-03157-4

16. Abrahamsson TR, Jakobsson HE, Andersson AF, Björkstén B, Engstrand L, Jenmalm MC. Low gut microbiota diversity in early infancy precedes asthma at school age. Clin Exp Allergy. 2014;44 (6):842-850. doi:10.1111/cea.12253

17. Chiu CY, Cheng ML, Chiang MH, et al. Gut microbial-derived butyrate is inversely associated with IgE responses to allergens in childhood asthma. Pediatr Allergy Immunol. 2019;30(7):689-697. doi:10.1111/pai.13096

18. Barcik W, Pugin B, Westermann $\mathrm{P}$, et al. Histamine-secreting microbes are increased in the gut of adult asthma patients. $J$ Allergy Clin Immunol. 2016;138(5):1491-1494.e7. doi:10.1016/j.jaci.2016. 05.049

19. Begley L, Madapoosi S, Opron K, et al. Gut microbiota relationships to lung function and adult asthma phenotype: a pilot study. $B M J$ Open Respir Res. 2018;5(1):e000324. doi:10.1136/bmjresp-2018000324

20. Edgar RC. UPARSE: highly accurate OTU sequences from microbial amplicon reads. Nat Methods. 2013;10(10):996-998. doi:10.1038/ nmeth.2604

21. Caporaso JG, Kuczynski J, Stombaugh J, et al. QIIME allows analysis of high-throughput community sequencing data. Nat Methods. 2010;7(5):335-336. doi:10.1038/nmeth.f.303

22. Zhang J, Zhang N, Liu YX, et al. Root microbiota shift in rice correlates with resident time in the field and developmental stage. Sci China Life Sci. 2018;61(6):613-621. doi:10.1007/s11427-0189284-4

23. Wang Q, Li F, Liang B, et al. A metagenome-wide association study of gut microbiota in asthma in UK adults. BMC Microbiol. 2018;18 (1):114. doi:10.1186/s12866-018-1257-x

24. Stokholm J, Blaser MJ, Thorsen J, et al. Maturation of the gut microbiome and risk of asthma in childhood. Nat Commun. 2018;9 (1):141. doi:10.1038/s41467-017-02573-2

25. Chen CC, Chen KJ, Kong MS, Chang HJ, Huang JL. Alterations in the gut microbiotas of children with food sensitization in early life. Pediatr Allergy Immunol. 2015;27(3):254-262. doi:10.1111/pai.12 522

26. Abdel-Aziz MI, Vijverberg SJH, Neerincx AH, Kraneveld AD, Maitland-van der Zee AH. The crosstalk between microbiome and asthma: exploring associations and challenges. Clin Exp Allergy. 2019;49(8):1067-1086. doi:10.1111/cea.13444 
27. Lee JJ, Kim SH, Lee MJ, et al. Different upper airway microbiome and their functional genes associated with asthma in young adults and elderly individuals. Allergy. 2019;74(4):709-719. doi:10.1111/all.13608

28. Maier L, Pruteanu M, Kuhn M, et al. Extensive impact of non-antibiotic drugs on human gut bacteria. Nature. 2018;555 (7698):623-628. doi:10.1038/nature25979

29. Singh RK, Chang HW, Yan D, et al. Influence of diet on the gut microbiome and implications for human health. J Transl Med. 2017;15(1):73. doi:10.1186/s12967-017-1175-y

30. Liang Z, Liu L, Zhao H, et al. A systemic inflammatory endotype of asthma with more severe disease identified by unbiased clustering of the serum cytokine profile. Medicine. 2016;95(25):e3774. doi:10.1097/MD.0000000000003774

31. Chung KF. Asthma phenotyping: a necessity for improved therapeutic precision and new targeted therapies. J Intern Med. 2016;279 (2):192-204. doi:10.1111/joim.12382

32. Ryan MP, Pembroke JT. Brevundimonas spp: emerging global opportunistic pathogens. Virulence. 2018;9(1):480-493. doi:10.1080/ 21505594.2017.1419116

33. Kowalska-Duplaga K, Gosiewski T, Kapusta P, et al. Differences in the intestinal microbiome of healthy children and patients with newly diagnosed crohn's disease. Sci Rep. 2019;9(1):18880. doi:10.1038/ s41598-019-55290-9

34. Arnold RJ, Massanari M, Lee TA, Brooks E. A review of the utility and cost effectiveness of monitoring Fractional Exhaled Nitric Oxide (FENO) in asthma management. Manag Care. 2018;27(7):34-41.

35. Nagase $H$. The role of fractional nitric oxide in exhaled breath (FENO) in clinical practice of asthma. Rinsho Byori. 2014;62 (12):1226-1233.

36. Fielding S, Pijnenburg M, De jongste JC, et al. Change in FEV1 and FENO measurements as predictors of future asthma outcomes in children. Chest. 2019;155(2):331-341. doi:10.1016/j.chest.2018.10.009

37. Durack J, Lynch SV, Nariya S, et al. Features of the bronchial bacterial microbiome associated with atopy, asthma, and responsiveness to inhaled corticosteroid treatment. J Allergy Clin Immunol. 2017;140(1):63-75. doi:10.1016/j.jaci.2016.08.055
38. Kho AT, Sharma S, Davis JS, et al. Circulating MicroRNAs: association with lung function in asthma. PLoS One. 2016;11(6):e0157998. doi:10.1371/journal.pone.0157998

39. Zhang Y, Wang Z, Ma T. Associations of genetic polymorphisms relevant to metabolic pathway of vitamin D3 with development and prognosis of childhood bronchial asthma. DNA Cell Biol. 2017;36 (8):682-692. doi:10.1089/dna.2017.3730

40. Li W, Dai W, Sun J, et al. Association of peroxisome proliferator-activated receptor-gamma gene polymorphisms and gene-gene interaction with asthma risk in a Chinese adults population. Int J Clin Exp Med. 2015;8(10):19346-19352.

41. Banno A, Reddy AT, Lakshmi SP, Reddy RC. PPARs: key regulators of airway inflammation and potential therapeutic targets in asthma. Nucl Recept Res. 2018;5:101306. doi:10.11131/2018/ 101306

42. Hämäläinen N, Nwaru BI, Erlund I, et al. Serum carotenoid and tocopherol concentrations and risk of asthma in childhood: a nested case-control study. Clin Exp Allergy. 2017;47(3):401-409. doi:10.1111/cea.12904

43. Lago JH, Toledo-Arruda AC, Mernak M, et al. Structure-activity association of flavonoids in lung diseases. Molecules. 2014;19 (3):3570-3595. doi:10.3390/molecules 19033570

44. Kawai M, Hirano T, Higa S, et al. Flavonoids and related compounds as anti-allergic substances. Allergol Int. 2007;56(2):113-123. doi:10.2332/allergolint.R-06-135

45. Morris CR, Poljakovic M, Lavrisha L, Machado L, Kuypers FA, Morris SM Jr. Decreased arginine bioavailability and increased serum arginase activity in asthma. Am J Respir Crit Care Med. 2004;170(2):148-153. doi:10.1164/rccm.200309-130 $4 \mathrm{OC}$

46. Yang M, Rangasamy D, Matthaei KI, et al. Inhibition of arginase I activity by RNA interference attenuates IL-13-induced airways hyperresponsiveness. J Immunol. 2006;177(8):5595-5603. doi:10. 4049/jimmunol.177.8.5595

\section{Publish your work in this journal}

The Journal of Asthma and Allergy is an international, peer-reviewed open-access journal publishing original research, reports, editorials and commentaries on the following topics: Asthma; Pulmonary physiology; Asthma related clinical health; Clinical immunology and the immunological basis of disease; Pharmacological interventions and new therapies. The manuscript management system is completely online and includes a very quick and fair peer-review system, which is all easy to use. Visit http://www.dovepress.com/testimonials.php to read real quotes from published authors. 